\title{
The eclipsing massive X-ray binary M 33 X-7: New X-ray observations and optical identification ${ }^{\star}$
}

\author{
W. Pietsch ${ }^{1}$, B. J. Mochejska ${ }^{2,3}$, Z. Misanovic ${ }^{1}$, F. Haberl ${ }^{1}$, M. Ehle ${ }^{4}$, and G. Trinchieri ${ }^{5}$ \\ 1 Max-Planck-Institut für extraterrestrische Physik, 85741 Garching, Germany \\ 2 Harvard-Smithsonian Center for Astrophysics, 60 Garden Street, Cambridge, MA 02138, USA \\ ${ }^{3}$ Hubble Fellow \\ 4 XMM-Newton Science Operations Centre, ESA, Villafranca del Castillo, PO Box 50727, 28080 Madrid, Spain \\ 5 Osservatorio Astronomico di Brera, via Brera 28, 20121 Milano, Italy
}

Received 16 July 2003 / Accepted 29 September 2003

\begin{abstract}
The eclipsing X-ray binary M 33 X-7 was in the field of view during several observations of our XMM-Newton M 33 survey and in the archival Chandra observation 1730 which cover a large part of the $3.45 \mathrm{~d}$ orbital period. We detect emission of M 33 X-7 during eclipse and a soft X-ray spectrum of the source out of eclipse that can best be described by bremsstrahlung or disk blackbody models. No significant regular pulsations of the source in the range $0.25-1000 \mathrm{~s}$ were found. The average source luminosity out of eclipse is $5 \times 10^{37} \mathrm{erg} \mathrm{s}^{-1}(0.5-4.5 \mathrm{keV})$. In a special analysis of DIRECT observations we identify as optical counterpart a B0I to O7I star of 18.89 mag in V which shows the ellipsoidal heating light curve of a high mass X-ray binary with the M $33 \mathrm{X}-7$ binary period. The location of the X-ray eclipse and the optical minima allow us to determine an improved binary period and ephemeris of mid-eclipse as HJD $(2451760.61 \pm 0.09) \pm N \times(3.45376 \pm 0.00021)$. The mass of the compact object derived from orbital parameters and the optical companion mass, the lack of pulsations, and the X-ray spectrum of M 33 X-7 may indicate that the compact object in the system is a black hole. M 33 X-7 would be the first detected eclipsing high mass black hole X-ray binary.
\end{abstract}

Key words. galaxies: individual: M 33 - X-rays: individuals: M 33 X-7 - X-rays: binaries - binaries: eclipsing

\section{Introduction}

M $33 \mathrm{X}-7$ (hereafter $\mathrm{X}-7$ ) was detected as a variable source with a luminosity brighter than $10^{38} \mathrm{erg} \mathrm{s}^{-1}$ in Einstein observations (Long et al. 1981; Markert \& Rallis 1983; Trinchieri et al. 1988a,b). Peres et al. (1989) suggested that the X-7 variability pattern can be explained by an eclipsing X-ray binary (XRB) with an orbital period of $1.7 \mathrm{~d}$ and an eclipse duration of $\sim 0.4 \mathrm{~d}$. This finding was the first identification of a close accreting binary system with an X-ray source in an external galaxy other than the Magellanic Clouds. It was confirmed combining Einstein observatory and first ROSAT data (Schulman et al. 1993, 1994). With the inclusion of more ROSAT and ASCA data (Larson \& Schulman 1997; Dubus et al. 1999, hereafter D99) the orbital period turned out to be twice as long. The shape of the eclipse could be described by a slow ingress $\left(\Delta \Phi_{\text {ingress }}=0.10 \pm 0.05\right)$, an eclipse duration of $\Delta \Phi_{\text {eclipse }}=0.20 \pm 0.03$, and a fast eclipse egress $\left(\Delta \Phi_{\text {egress }}=0.01 \pm 0.01\right)$ with an ephemeris for the mideclipse time of HJD $2448631.5 \pm 0.1+N \times(3.4535 \pm 0.0005)$.

\footnotetext{
Send offprint requests to: W. Pietsch, e-mail: wnp@mpe.mpg. de

* This work is based on observations obtained with XMM-Newton, an ESA Science Mission with instruments and contributions directly funded by ESA Member States and the USA (NASA).
}

In addition, D99 discovered evidence for a $0.31 \mathrm{~s}$ pulse period. The orbital period, pulse period and observed X-ray luminosity are remarkably similar to those of the Small Magellanic Cloud neutron star XRB SMC X-1 (Liu et al. 2000). However, if the pulse period of $\mathrm{X}-7$ can not be confirmed, the source could also resemble high mass black hole XRBs (BHXB) like LMC X-1 or LMC X-3. It would be the first eclipsing object within this rare class of XRBs.

The position of $\mathrm{X}-7$ correlates with the dense O-B association HS13 (Humphreys \& Sandage 1980) and therefore no individual counterpart could be identified based on position only. However, its location in HS13 is consistent with the expectation of a massive companion. As D99 point out, the optical counterpart is likely to show ellipsoidal and/or X-ray heating variations (Tjemkes et al. 1986) which can be used for the optical identification.

Variable optical sources within M 33 were systematically searched for in the DIRECT project (see e.g. Mochejska et al. 2001b, hereafter M01b). Many eclipsing binaries, Cepheids, and other periodic, possibly long-period or nonperiodic variables were detected. X-7 is located in DIRECT field M 33B. The variability of the optical counterpart was not detected in the previous analysis due to the limitations of the variable search strategy for such small amplitude variables in crowded regions. 
Table 1. M 33 X-7 observations with the observatories XMM-Newton (proposal numbers 010264 and 014198) and Chandra (1730). Besides observation 0102642101, where M 33 X-7 was only in the field of view of the MOS detectors, for XMM-Newton we give EPIC PN count rates, hardness ratios and luminosities. For the low state observations, no hardness ratio could be determined.

\begin{tabular}{ccccccccc}
\hline \hline Obs. id. & Obs. dates & $\begin{array}{c}\text { Elapse time } \\
(\mathrm{ks})\end{array}$ & $\begin{array}{c}R_{\text {ext }} \\
\left({ }^{\prime \prime}\right)\end{array}$ & $\begin{array}{c}\text { Count rate } \\
\left(\mathrm{ct} \mathrm{ks}^{-1}\right)\end{array}$ & HR & $\begin{array}{c}L_{\mathrm{X}}^{* * *} \\
\left(10^{37} \mathrm{erg} \mathrm{s}^{-1}\right) \\
(7)\end{array}$ & $\begin{array}{c}\text { Offax } \\
\left({ }^{\prime}\right)\end{array}$ & $\begin{array}{c}\text { Binary phase } \\
(8)\end{array}$ \\
\hline 0102641201 & $2000-08-02$ & 13.1 & 30.0 & $172 \pm 4$ & $1.40 \pm 0.38^{\dagger}$ & 8.7 & 10.4 & $0.54-0.58$ \\
0102640401 & $2000-08-02$ & 13.1 & 40.0 & $163 \pm 5$ & $1.27 \pm 0.23^{\dagger}$ & 8.5 & 10.4 & $0.61-0.66$ \\
0102640101 & $2000-08-04$ & 13.3 & 22.5 & $4.0 \pm 1.2$ & & 0.2 & 8.3 & $0.11-0.16$ \\
0102640501 & $2001-07-05$ & 11.8 & 45.0 & $57 \pm 2$ & $1.27 \pm 0.30$ & 8.3 & 7.1 & $0.33-0.36$ \\
0102640601 & $2001-07-05$ & $8.2^{+}$ & 35.0 & $75 \pm 5$ & $1.09 \pm 0.45$ & 6.0 & 15.7 & $0.23-0.26$ \\
0102640701 & $2001-07-05$ & 11.7 & 35.0 & $54 \pm 2$ & $1.10 \pm 0.26$ & 7.8 & 9.3 & $0.28-0.31$ \\
0102541101 & $2001-07-08$ & 12.5 & 30.0 & $5.1 \pm 1.7$ & & 0.3 & 11.1 & $0.01-0.05$ \\
0102642101 & $2002-01-25$ & $12.3^{*}$ & 35.0 & $115 \pm 3^{*}$ & $1.61 \pm 0.35^{*}$ & 8.5 & 13.1 & $0.25-0.29$ \\
0102642301 & $2002-01-27$ & 12.3 & 35.0 & $316 \pm 6$ & $1.17 \pm 0.20$ & 7.0 & 0.9 & $0.81-0.85$ \\
0141980501 & $2003-01-22$ & 8.1 & 35.0 & $114 \pm 10$ & $1.20 \pm 0.15$ & 9.0 & 8.3 & $0.18-0.21$ \\
0141980601 & $2003-01-23$ & 13.6 & 37.5 & $153 \pm 4$ & $1.23 \pm 0.28$ & 7.3 & 10.4 & $0.46-0.50$ \\
0141980701 & $2003-01-24$ & 13.7 & 35.0 & $164 \pm 6$ & $1.12 \pm 0.34$ & 7.3 & 10.4 & $0.52-0.56$ \\
0141980801 & $2003-02-12$ & 10.2 & 35.0 & $221 \pm 6$ & $1.16 \pm 0.28$ & 8.6 & 8.3 & $0.20-0.23$ \\
1730 & $2000-12-07$ & 52.0 & 15.0 & $100 \pm 2$ & $1.89 \pm 0.09$ & 6.3 & 8.6 & $0.49-0.65$ \\
\hline
\end{tabular}

Notes and references:

+ : M 33 X-7 only in PN field of view.

* : M 33 X-7 only in MOS field of view, integrated MOS count rate, hardness ratio and luminosity given.

$\dagger$ : The on average higher HR during observations 0102641201 and 0102640401 compared to the other observations reflects the difference between thick and medium filter and most likely not a change of the spectrum of M 33 X-7.

${ }^{* *}$ : raw count rate and luminosity in the $0.5-4.5 \mathrm{keV}$ band assuming the best fitting thermal bremsstrahlung spectrum (see Table 3 ) corrected for a Galactic foreground absorption of $N_{\mathrm{H}}=6.38 \times 10^{20} \mathrm{~cm}^{-2}$ (Dickey \& Lockman 1990) and a distance of M $33 \mathrm{of} 795 \mathrm{kpc}$ (van den Bergh 1991), which we use throughout the paper.

As a follow-up of our study of the X-ray source population of M 33 based on all archival ROSAT observations (Haberl \& Pietsch 2001, hereafter HP01), we planned a deep XMM-Newton raster survey of M 33 based on 22 Telescope Scientist guaranteed time (proposal no 010264) and AO2 (proposal no 014198) observations, each with a duration of about $10 \mathrm{ks}$ (for first results see Pietsch et al. 2003). X-7 was covered in 13 of these observations at varying off-axis angles and covering different orbital phases.

In this paper we report on time and spectral variability of X-7 within the XMM-Newton raster survey. We add results from an archival Chandra observation, which covered the source, and a dedicated timing analysis of the DIRECT data of the HS13 region.

\section{X-ray observations and results}

For the detailed analysis of X-7 we mostly used data from XMM-Newton EPIC. From the M 33 observations in the Chandra archive only the observation with identification no 1730 covered the $\mathrm{X}-7$ field. Table 1 summarizes the observation identifications (Col. 1), observation dates (2), elapse time (3), extraction radius $R_{\text {ext }}$ used for count rates, light curves, and spectra (4), X-7 raw count rates in the $0.5-4.5 \mathrm{keV}$ band (5), hardness ratios (6), and luminosities in the $0.5-4.5 \mathrm{keV}$ band (7). For the XMM-Newton observations, values in Cols. 4 to 7 correspond to the EPIC $\mathrm{PN}$ detector if not indicated differently, since it gives about twice the number of photons than the EPIC MOS cameras. Off axis angle of $\mathrm{X}-7$ (8) and phase coverage within the binary orbit according to the ephemeris of D99 (9) are also given. As hardness ratio (HR) we use the ratio of the counts in the $1.2-3.0 \mathrm{keV}$ band to the counts in the $0.5-$ $1.2 \mathrm{keV}$ band. Luminosities were determined from thermal bremsstrahlung spectra (see Sect. 2.3). During observations 0102640101 and $0102641101 \mathrm{X}-7$ was in low state and source statistics did not allow us to derive hardness ratios. For these observations we assumed that the source spectrum was similar to the spectrum during observation 0102642301 and scaled the source luminosity from the vignetting corrected count rates.

In the XMM-Newton observations (Jansen et al. 2001) the EPIC PN and MOS instruments (Strüder et al. 2001; Turner et al. 2001) were mostly operated in the full frame mode resulting in a time resolution of $73.4 \mathrm{~ms}$ and $2.6 \mathrm{~s}$, respectively. Only for the first two observations in Table 1, the PN detector was operated in the extended full frame mode (time resolution $200 \mathrm{~ms}$ ) and during observation 0102640101 the MOS detectors were operated in the small window mode $(0.3 \mathrm{~s}$ time resolution for the inner CCDs). The medium filter was in front of the EPIC cameras in all but the first two observations which were performed with the thick filter. We used all EPIC instruments for imaging, position determination and for the timing 
and spectral investigations of $\mathrm{X}-7$. In most of the observations the source is located at high off-axis angle (see Table 1), and could be outside of the field of view in some of the cameras. Also, the cameras normally cover different times. The XMM-Newton point spread function (PSF) required extraction radii $R_{\text {ext }}$ larger than 22 .' 5 to encircle $>80 \%$ of the source photons. Depending on the location of the source, counts could be missing due to CCD gaps. Many of the XMM-Newton observations suffer from times of high particle background. To be able to also use these times to cover as much as possible of the X-7 binary orbit, we restricted the energy band for light curve and hardness ratio analysis to $0.5-3.0 \mathrm{keV}$ where the source is brightest.

Four Chandra ACIS observations (Weisskopf et al. 2000) of M 33 were obtained from the Chandra Data Archive (http: //asc.harvard.edu/cgi-gen/cda). However, only the ACIS I observation 1730 (see Table 1) covered the X-7 field. The instrument was operated in the full frame mode $(3.2 \mathrm{~s}$ time resolution). $\mathrm{X}-7$ is positioned in the outer corner of the front-illuminated CCD chip I 2 during the observation.

The deep space orbits of the satellites XMM-Newton and Chandra led to long continuous observation times of X-7. The low earth orbits of the Einstein, ROSAT, ASCA and BeppoSAX observatories on the other hand, led to observations split in many short intervals of typically less than $1500 \mathrm{~s}$.

The data analysis was performed using tools in the SAS v5.4.0, CIAO v2.3, EXSAS/MIDAS 1.2/1.4, and FTOOLS v5.2 software packages, the imaging application DS9 v2.1b4, the timing analysis package XRONOS v5.19 and spectral analysis software XSPEC v11.2.

For the time variability investigations all $\mathrm{X}-7$ event times were corrected to solar system barycenter arrival times.

\subsection{Time variability}

The X-ray light curve of $\mathrm{X}-7$ was sampled with a time resolution of $1000 \mathrm{~s}$ for EPIC PN and with $2000 \mathrm{~s}$ for MOS. While we give raw count rate in Table 1, the light curves in Figs. 1 and 4 are corrected for vignetting and EPIC MOS count rates for the difference in instrument efficiency compared to EPIC PN (i.e. increased by a factor of 2.5). The vignetting corrections for far off-axis positions is up to a factor of 3.5 and may include systematic errors of up to $20 \%$. The source extraction radii chosen assure that more than $80 \%$ of the PSF are covered. EPIC PN as well as MOS show X-7 at low intensity for XMM-Newton observations 0102640101 and 0102641101 which covered binary phase 0.01 to 0.16 according to the ephemeris of D99. During the other XMM-Newton observations (covering binary phase 0.18 to 0.85 according to the ephemeris of D99, see Figs. 1 and 4), the source was in high state. The high state intensity did not vary by more than $\pm 20 \%$ between the observations. Within the observations the intensities changed by up to $30 \%$ on time scales as short as $1000 \mathrm{~s}$ (see Fig. 1). No significant variability in hardness ratio was detected between the observations. However, there seems to be variability on shorter time scales. Some of the observations suffer from strong particle background flares which show vignetting effects by the

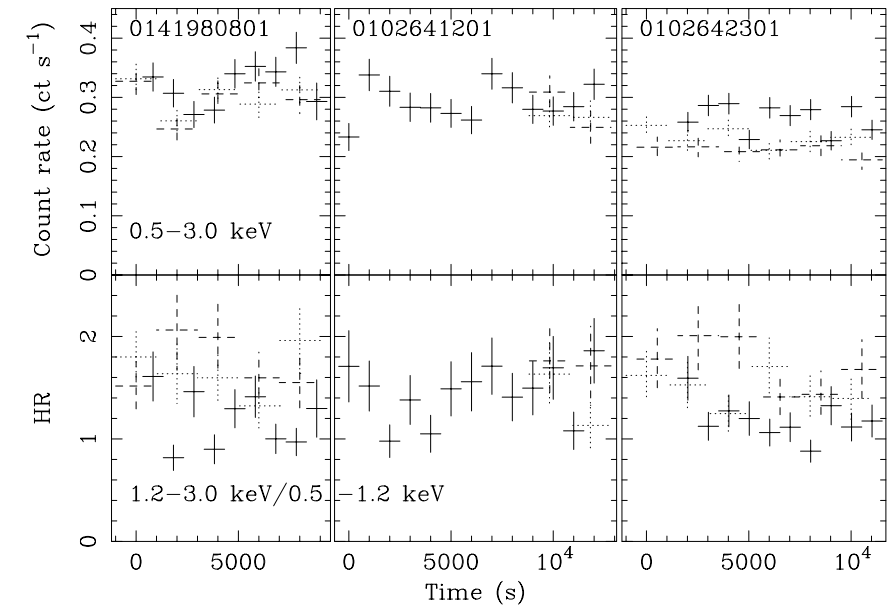

Fig. 1. XMM-Newton EPIC light curves and hardness ratio of M 33 X-7 during observations 0141980801, 0102641201, and 102642301 with time zero corresponding to HJD 2452683.14827 , $2451758.79473,2452301.91542$, respectively (solar system barycenter corrected). Count rates were corrected for vignetting. EPIC MOS rates were scaled by a factor of 2.5 to approximately correct for the difference in instrument efficiency compared to EPIC PN. EPIC PN, MOS1 and MOS2 data are marked with solid, dotted, and dashed error bars. The light curves cover orbital phases $0.20-0.23$, 0.54-0.58, and 0.81-0.85 (left to right, ephemeris of D99).

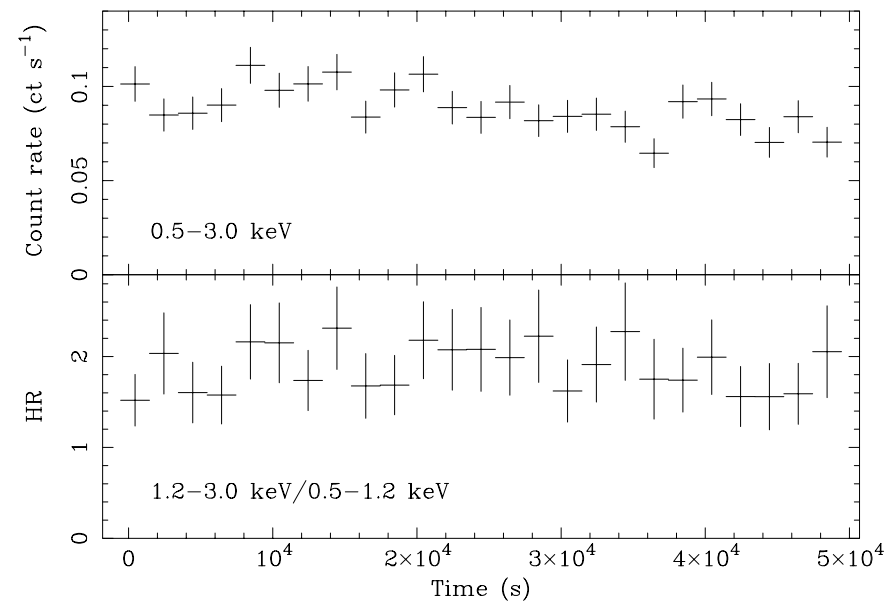

Fig. 2. Chandra ACIS I light curve and hardness ratio of M $33 \mathrm{X}-7$ during observation 1730 integrated over 2000 s. Time zero corresponds to HJD 2451737.88019 (solar system barycenter corrected). The light curves cover orbital phases 0.49-0.65 (ephemeris of D99).

telescope mirrors. We did not exclude these times from the light curves but tried to correct by subtracting this variable background determined as close to $\mathrm{X}-7$ as allowed by CCD configuration and emission from other nearby sources. Our selection of the background regions should minimize spurious effects on source count rate and hardness ratio. Nevertheless, residual effects may explain the count rate increase in observation 0141980701 around binary phase 0.55 seen in Fig. 4.

The light curve for the Chandra observation 1730 shows the source out of eclipse. While the count rate in the $0.5-3.0 \mathrm{keV}$ band decreased during the observation by about $20 \%$ and there is also variability down to the 

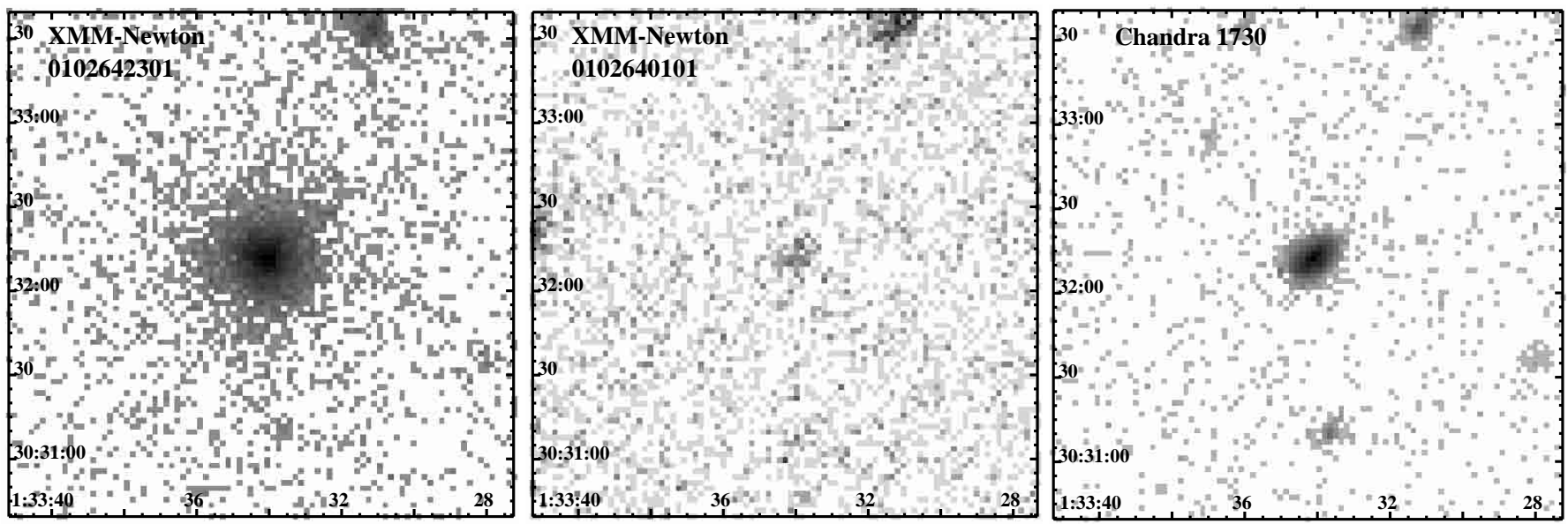

Fig. 3. Logarithmically-scaled, grey-scale images of the M 33 X-7 region of the XMM-Newton EPIC observations 0102642301, 0102640101, and Chandra ACIS I observation 1730 (from left to right). The images (RA, Dec J2000.0) were accumulated with a pixel size of 2", in the $0.5-4.5 \mathrm{keV}$ band. EPIC PN and MOS images were selected for times of low background and added. Integration times for observation 0102642301 are 9.0, 12.1, and 12.2 ks for PN, MOS1, and MOS2, respectively, and the maximum in the image is 193 counts per pixel. The corresponding numbers for observation 0102640101 are 11.7, 10.6, and $12.4 \mathrm{ks}$ and 12 counts per pixel. In this image M $33 \mathrm{X}-7$ is close to a CCD gap in PN and there is no MOS exposure in the upper left corner of the image. For the Chandra image the integration time was $49.4 \mathrm{ks}$ and the maximum in the image is 919 counts per pixel. M $33 \mathrm{X}-7$ is the source in the center of the images and was in a low state (eclipse) during observation 0102640101 . HP01 X67 is the source $\sim 1.5$ to the NNW of M 33 X-7.

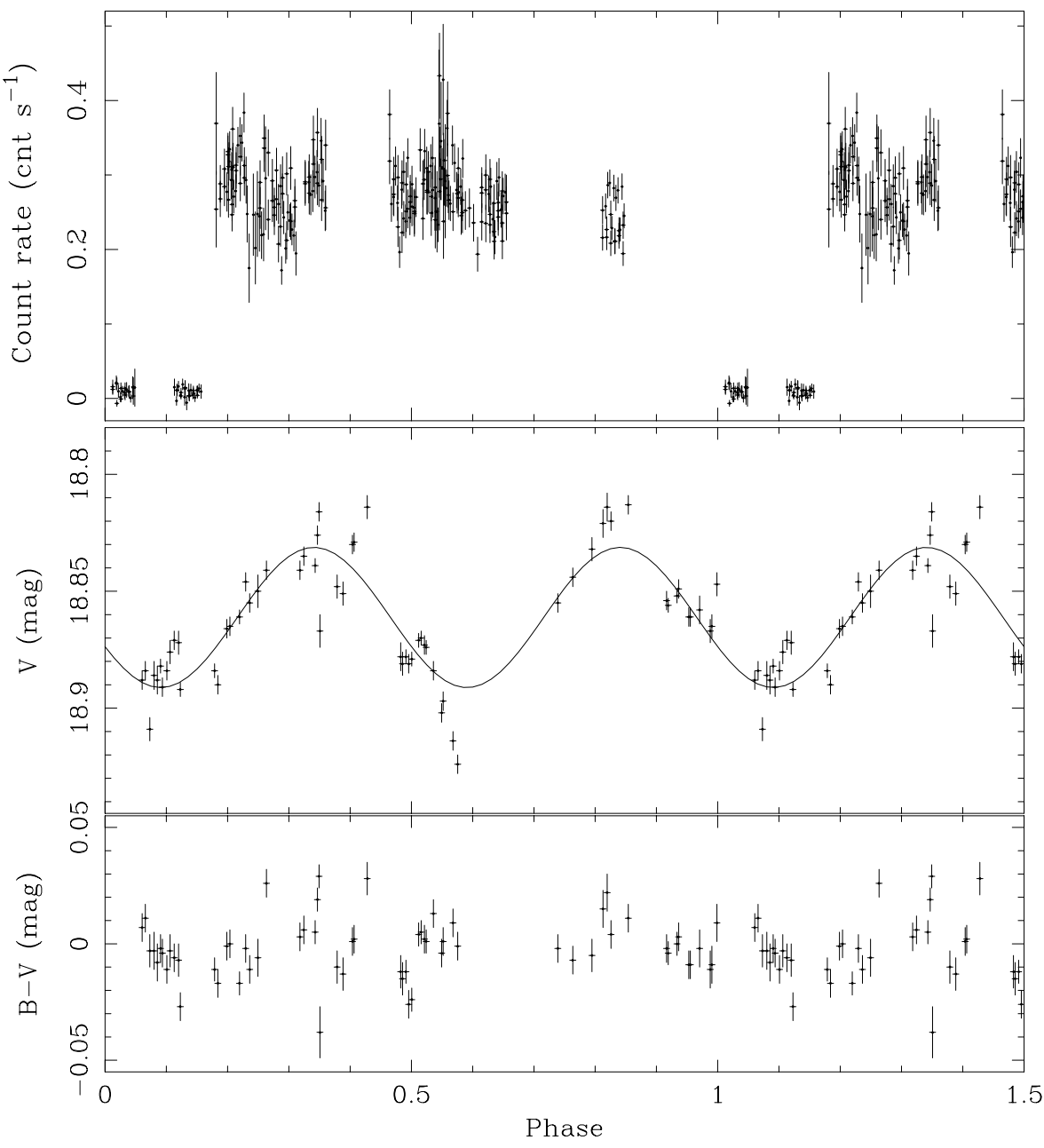

Fig. 4. Light curve of the XRB M $33 X-7$ in the $0.5-3.0 \mathrm{keV}$ band and in optical $V$ and $B-$ $V$ folded over the $3.45 \mathrm{~d}$ orbital period using ephemeris of D99. Data for phase 0. to 0.5 are repeated at 1.0 to 1.5 for clarity. Added is a double-sinusoidal approximation to the $V$-data (full line, see text). 
sampling time scale of $2000 \mathrm{~s}$, the hardness ratio did not change significantly (Fig. 2).

One has to keep in mind that the EPIC MOS count rates and HRs of $\mathrm{X}-7$ can not directly be compared to the PN one as they originate from a CCD with a different energy response function. The same is even more true if one wants to compare Chandra and XMM-Newton count rates and HRs.

Figure 3 shows images ( $3^{\prime}$ to a side) of the $X-7$ area in the $0.5-4.5 \mathrm{keV}$ band during the high and low state (XMM-Newton EPIC cameras are combined). X-7 is clearly detected also during the low state ( $\sim 3 \%$ of the average high state emission).

We searched for pulsations within the bright state XMM-Newton low background observations and the Chandra observation in the frequency range $10^{-3}-4 \mathrm{~Hz}$ following the Rayleigh $Z_{n}^{2}$ method (Buccheri et al. 1983) as described in Haberl \& Zavlin (2002). No signal was found with more than $94 \%(<2 \sigma)$ confidence level. This includes the previously suggested period of $0.31 \mathrm{~s}$ (D99) although the time resolution of $0.073 \mathrm{~s}$ of the PN detector in the full frame mode may not be sufficient to clearly detect such a short period.

\subsection{Improved position}

HP01 give the position of $X-7$ with a $90 \%$ error radius of 2 .'0 which is fully determined by the assumed remaining systematic error of 2 ". 0 . The much higher number of photons detected with the Chandra ACIS I detectors and the good PSF even at an off-axis angle of 8.6 allow us to determine a significantly improved source position in observation 1730. Systematic errors can be reduced by adjusting the positions using the well determined radio position of a supernova remnant (SNR) close to X-7 (source 57 in Gordon et al. 1999) which is also detected in X-rays (X67 in HP01), the X-ray source $\sim 1.5$ to the NNW of $X-7$ in Fig. 3. We get a significantly improved position for X-7: $\alpha=01^{\mathrm{h}} 33^{\mathrm{m}} 34^{\mathrm{s}} .21, \delta=+30^{\circ} 32^{\prime} 11^{\prime \prime} .7$ (J2000), with a remaining error radius of 0.3 mainly determined from the statistical uncertainty of the X67 position.

Several XMM-Newton EPIC observations cover X-7 and in addition X67. The integration times of the individual observations are much shorter than for the Chandra observation and the XMM-Newton PSF is worse. XMM-Newton determined positions of $\mathrm{X}-7$ have individual error radii of 1 .' 0 or greater for the PN and MOS cameras and are within the errors consistent with the Chandra position. See Fig. 5 for an overlay of the improved position on a deep optical $V$-band image.

\subsection{Energy spectra}

Spectra for the low background XMM-Newton EPIC observation 0102642301 and for the Chandra observation were approximated with simple spectral models (see Table 2). Individual EPIC PN and MOS spectra were simultaneously fitted with the same model parameters correcting for vignetting and the fraction of the PSF covered by the source extraction area. Energy independent normalization factors for each spectrum separately take into account possible differences in source coverage. Model components were the fixed Galactic foreground absorption plus additional absorption within M 33 or in the immediate surrounding of the source using XSPEC model

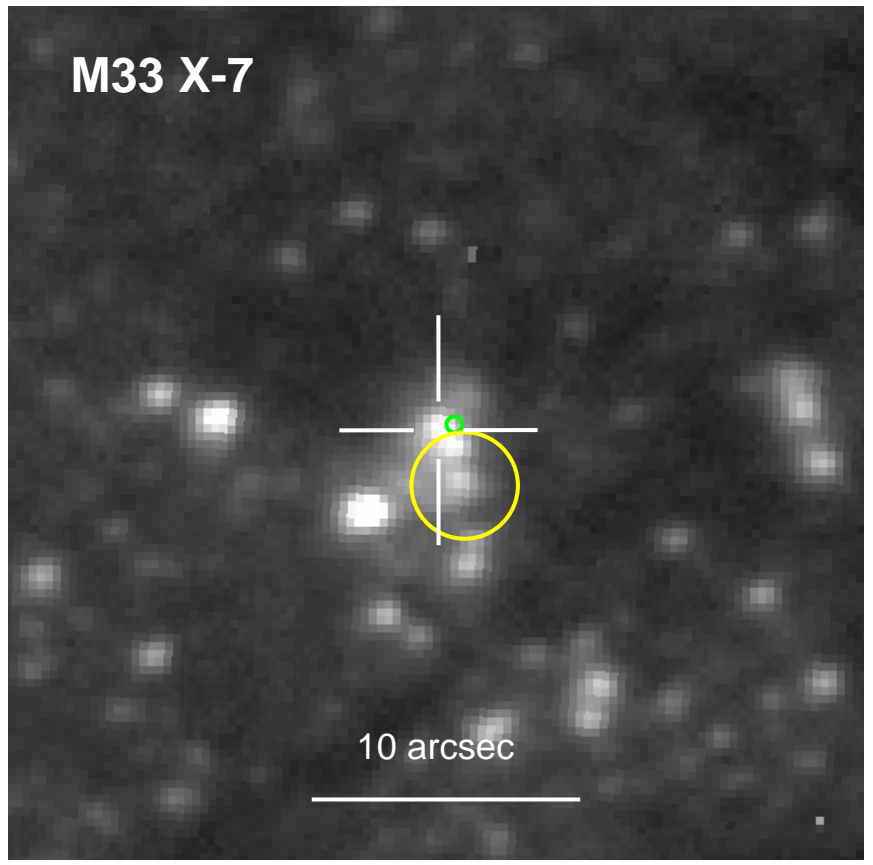

Fig. 5. Position of M $33 \mathrm{X}-7$ is shown on a $V$ image of the DIRECT survey. The ROSAT position and error from HP01 is marked by the big circle, Chandra position by the small circle. The optical identification, i.e. the star showing the $3.4 \mathrm{~d}$ variability, is marked by the cross hair.

component WABS. A thin thermal (MEKAL) model could be rejected due to the high $\chi^{2} / v$, and also a power law (POWL) model still shows large residuals. Bremsstrahlung (BREMS) and also variable temperature disk blackbody (DISKBB) models on the other hand yield acceptable $\chi^{2} / v$ values. We used the latter models to fit all high state observations of X-7 (see Table 3). For all observations, absorbed BREMS or DISKBB models give acceptable fits. The BREMS model needs comparably high absorption outside the Galaxy. The model temperatures are the same within the errors. For the DISKBB model, on the other hand, the additional absorption is very low or absent and inner disk temperatures also don't vary significantly.

\section{Optical observations and results}

\subsection{Observations}

The data were obtained as part of the DIRECT project ${ }^{1}$ (Mochejska et al. 2001a,c, M01b) at the Kitt Peak National Observatory $^{2} 2.1 \mathrm{~m}$ telescope during two separate runs, from September 29th to October 5th, 1999 and from November 1st to 7 th, 1999. The telescope was equipped with a Tektronix $2048 \times$ 2048 CCD (T2KA camera) having a pixel scale $0.305^{\prime \prime} /$ pixel. We collected $74 \times 600 \mathrm{~s}$ exposures in the $V$ filter and $30 \times 600 \mathrm{~s}$ in $B$. The exposure times varied slightly to compensate for the changes of seeing conditions. The typical seeing was 1 '.5.

\footnotetext{
${ }^{1}$ For information on the DIRECT project see http: //cfa-www.harvard.edu/ kstanek/DIRECT/.

${ }^{2}$ Kitt Peak National Observatory is a division of NOAO, which are operated by the Association of Universities for Research in Astronomy, Inc. under cooperative agreement with the National Science Foundation.
} 
Table 2. Spectral modeling results for M 33 X-7 for the on-axis XMM-Newton EPIC observation 0102641201 (all EPIC instruments fitted together) and the Chandra ACIS I observation 1730. The degradation of the ACIS was taken into account using model ACISABS in XSPEC. For each instrument, we give the effective integration time $T_{\text {int }}$ and the raw count rate. The number of energy bins reduced by the number of free parameters defines the degrees of freedom $v$. In the case of $\chi^{2} / v \leq 2.0,90 \%$ errors are given.

\begin{tabular}{|c|c|c|c|c|c|c|c|c|c|c|}
\hline Observation & Inst. & $\begin{array}{l}T_{\text {int }} \\
(\mathrm{ks})\end{array}$ & $\begin{array}{l}\text { Raw count rate } \\
\qquad\left(10^{-2} \mathrm{ct} \mathrm{s}^{-1}\right)\end{array}$ & $v$ & Model $^{*}$ & $\begin{array}{r}N_{\mathrm{HM} 33}^{* *} \\
\left(10^{20} \mathrm{~cm}^{-2}\right)\end{array}$ & $\Gamma$ & $\begin{array}{r}k T \\
(\mathrm{keV})\end{array}$ & $\begin{array}{r}L_{\mathrm{X}}^{* * *} \\
\left(10^{37} \mathrm{erg} \mathrm{s}^{-1}\right)\end{array}$ & $\chi^{2} / v$ \\
\hline XMM-Newton & $\mathrm{PN}$ & 9.0 & $32.0 \pm 0.6$ & 119 & POWL & $19.3_{-2.8}^{+3.1}$ & $2.24_{-0.09}^{+0.09}$ & & $6.9_{-0.6}^{+0.6}$ & 0.95 \\
\hline EPIC & MOS1 & 12.1 & $10.5 \pm 0.3$ & & BREMS & $8.8_{-2.0}^{+2.0}$ & & $2.93_{-0.27}^{+0.32}$ & $7.0_{-0.5}^{+0.5}$ & 0.79 \\
\hline \multirow[t]{2}{*}{0102642301} & MOS2 & 12.2 & $9.2 \pm 0.3$ & & THPL & $2.3_{-1.4}^{+1.5}$ & & $4.7_{-0.2}^{+0.4}$ & $7.0_{-0.3}^{+0.3}$ & 1.47 \\
\hline & & & & & DISKBB & $<0.8$ & & $0.96_{-0.03}^{+0.04}$ & $7.1_{-0.8}^{+1.0}$ & 0.87 \\
\hline Chandra & ACIS I & 49.4 & $10.2 \pm 0.2$ & 101 & POWL & $28.4_{-3.8}^{+4.1}$ & $2.33_{-0.08}^{+0.11}$ & & $6.2_{-0.6}^{+0.6}$ & 1.23 \\
\hline \multirow[t]{3}{*}{1730} & & & & & BREMS & $15.3_{-2.6}^{+2.8}$ & & $2.82_{-0.24}^{+0.27}$ & $6.3_{-0.4}^{+0.4}$ & 0.98 \\
\hline & & & & & THPL & 7.1 & & 4.7 & 6.3 & 2.1 \\
\hline & & & & & DISKBB & $3.2_{-2.3}^{+2.4}$ & & $1.01_{-0.05}^{+0.04}$ & $6.5_{-1.1}^{+1.3}$ & 0.97 \\
\hline
\end{tabular}

*: $\quad$ THPL $=$ thin thermal Plasma with solar abundance (XSPEC model MEKAL), BREMS $=$ thermal bremsstrahlung,

POWL $=$ power law, DISKBB $=$ disk blackbody.

** : Absorption exceeding the fixed Galactic foreground.

***: In the $0.5-4.5 \mathrm{keV}$ band, corrected for Galactic absorption, corrected for extraction radii and vignetting.

Table 3. Spectral modeling results for the remaining out of eclipse XMM-Newton EPIC observations of M $33 \mathrm{X}-7$ using thermal bremsstrahlung and disk blackbody spectra. In the last row, five EPIC PN spectra of low background observations ( $\Sigma$ EPIC PN; combining 0102640501, 0102640701, 0102642301, 0141980601, and 0141980801) are simultaneously fitted. Model parameters are given with 90\% errors (see Table 2).

\begin{tabular}{|c|c|c|c|c|c|c|c|}
\hline \multirow[t]{2}{*}{ Observation } & \multirow[t]{2}{*}{$v$} & \multicolumn{2}{|c|}{ Thermal bremsstrahlung } & \multicolumn{3}{|c|}{ Disk blackbody } & \multirow[b]{2}{*}{$\chi^{2} / v$} \\
\hline & & $\begin{array}{r}N_{\mathrm{HM} 33} \\
\left(10^{20} \mathrm{~cm}^{-2}\right)\end{array}$ & $\begin{array}{r}k T \\
(\mathrm{keV})\end{array}$ & $\chi^{2} / v$ & $\begin{array}{r}N_{\mathrm{HM} 33} \\
\left(10^{20} \mathrm{~cm}^{-2}\right)\end{array}$ & $\begin{array}{r}k T \\
(\mathrm{keV})\end{array}$ & \\
\hline 0102641201 & 53 & $16.0_{-3.3}^{+3.3}$ & $2.16_{-0.22}^{+0.26}$ & 0.79 & $4.2_{-2.8}^{+3.1}$ & $0.87_{-0.05}^{+0.06}$ & 0.96 \\
\hline 0102640401 & 68 & $12.3_{-3.0}^{+3.1}$ & $2.66_{-0.27}^{+0.23}$ & 1.05 & $<2.6$ & $0.99_{-0.06}^{+0.04}$ & 1.20 \\
\hline 0102640501 & 53 & $13.5_{-3.6}^{+3.8}$ & $2.72_{-0.33}^{+0.39}$ & 1.00 & $<4.6$ & $0.99_{-0.06}^{+0.06}$ & 0.99 \\
\hline $0102640601^{+}$ & 18 & $11.4_{-9.3}^{+10.4}$ & $2.87_{-1.26}^{+4.29}$ & 0.88 & $<11.7$ & $0.90_{-0.23}^{+0.25}$ & 0.99 \\
\hline 0102640701 & 41 & $10.8_{-4.0}^{+4.3}$ & $2.90_{-0.47}^{+0.61}$ & 0.91 & $<3.3$ & $0.98_{-0.07}^{+0.06}$ & 0.93 \\
\hline $0102642101^{*}$ & 30 & $14.4_{-4.6}^{+5.1}$ & $3.00_{-0.61}^{+0.87}$ & 0.97 & $<8.4$ & $0.97_{-0.10}^{+0.11}$ & 0.97 \\
\hline 0141980501 & 45 & $12.7_{-4.3}^{+4.6}$ & $2.54_{-0.39}^{+0.48}$ & 0.61 & $<4.8$ & $0.94_{-0.08}^{+0.07}$ & 0.79 \\
\hline 0141980601 & 75 & $17.0_{-2.7}^{+2.9}$ & $2.14_{-0.18}^{+0.21}$ & 1.25 & $4.8_{-2.4}^{+2.6}$ & $0.88_{-0.05}^{+0.05}$ & 1.15 \\
\hline 0141980701 & 87 & $9.4_{-3.9}^{+4.1}$ & $2.83_{-0.58}^{+0.89}$ & 1.06 & $<3.9$ & $0.90_{-0.09}^{+0.08}$ & 1.04 \\
\hline 0141980801 & 80 & $14.7_{-2.8}^{+3.9}$ & $2.13_{-0.17}^{+0.20}$ & 1.01 & $<4.4$ & $0.89_{-0.05}^{+0.04}$ & 0.92 \\
\hline$\Sigma$ EPIC PN & 251 & $11.6_{-1.5}^{+1.6}$ & $2.47_{-0.15}^{+0.17}$ & 1.07 & $<1.7$ & $0.94_{-0.03}^{+0.03}$ & 1.04 \\
\hline
\end{tabular}

\subsection{Data reduction and calibration}

The preliminary processing of the CCD frames was performed with the standard routines in the IRAF ccdproc package. ${ }^{3}$ The data were corrected for CCD non-linearity at this stage, as described by Mochejska et al. (2001a).

Photometry was extracted using the ISIS image subtraction package (Alard \& Lupton 1998; Alard 2000). A brief outline of the method is presented here. For a more detailed description

${ }^{3}$ IRAF is distributed by the National Optical Astronomy Observatories, which are operated by the Association of Universities for Research in Astronomy, Inc., under cooperative agreement with the NSF. of the reduction procedure the reader is referred to Mochejska et al. (2001a).

The ISIS reduction procedure consists of the following steps: (1) transformation of all frames to a common $(x, y)$ coordinate grid; (2) construction of a reference image from several best exposures; (3) subtraction of each frame from the reference image and (4) extraction of profile photometry from the subtracted images.

All computations were performed with the frames internally subdivided into four sections ( sub_x $_{\mathrm{s}}$ sub_y=2). Differential brightness variations of the background were fit with a second degree polynomial $($ deg_bg=2). A convolution kernel varying quadratically with position was used 

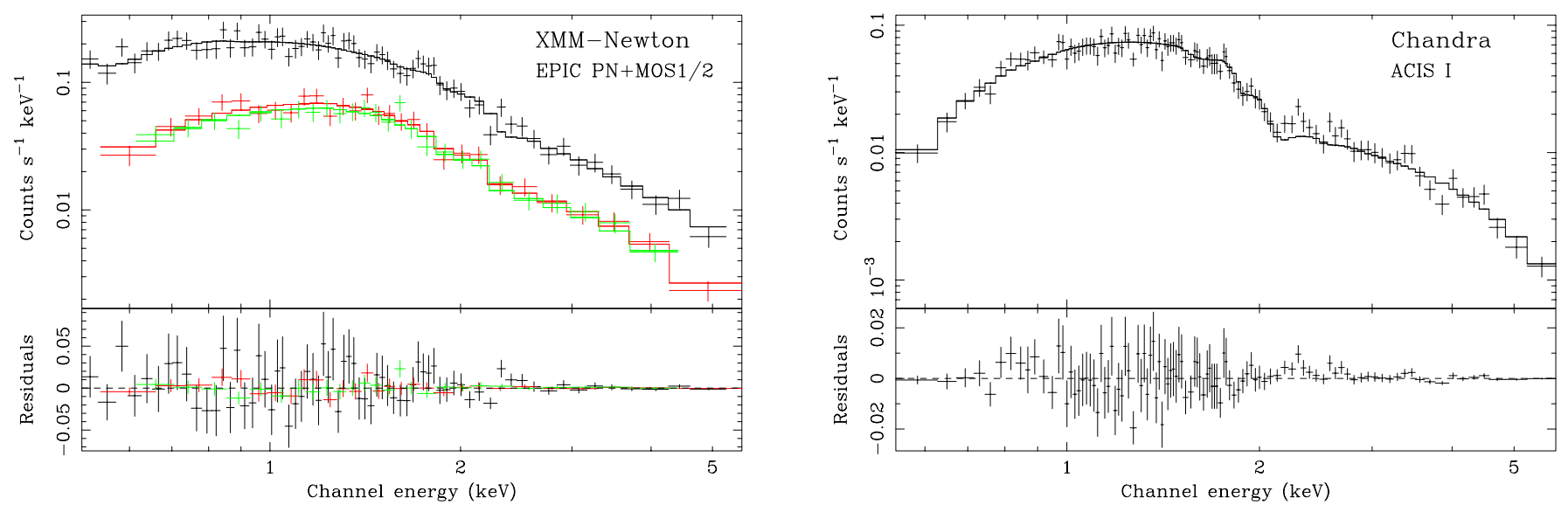

Fig. 6. M 33 X-7 spectrum of XMM-Newton EPIC PN observation 0102641201 (left) and of Chandra ACIS I observation 1730 (right). Data and best fitting bremsstrahlung model are shown in the upper panels, residuals between data and model below. For the model parameters see Table 2 .

(deg_spatial=2). The PSF width (psf_width) was set to 15 pixels. We used a photometric radius (radphot) of 3 pixels.

Due to residual non-linearity, our photometry could not be calibrated from observations of standard stars. The coefficients for the color terms of the transformation were derived from the comparison of our NGC 6791 photometry with the data from the KPNO 0.9m telescope (Kaluzny \& Udalski 1992). The offsets were determined relative to 735 stars above $V=20 \mathrm{mag}$ from the DIRECT catalog of stellar objects in M 33 (Macri et al. 2001). The calibration coefficients can be found in M01b.

The transformation from rectangular to equatorial coordinates was derived using 894 transformation stars with $V<$ 19.5 mag from the DIRECT catalog of stellar objects in M 33 (Macri et al. 2001). The average difference between the catalog and the computed coordinates for the transformation stars was $0 . ' 06$ in right ascension and $0 . ' 06$ in declination.

\subsection{Results}

We have searched within a radius of $36^{\prime \prime}$ around the SIMBAD position of $\mathrm{X}-7$ for stars with a period of around $3.45 \mathrm{~d}$. From the 715 stars examined only one was a binary with a period of about 3.46-3.48 days. It is a star at position $\alpha=$ $01^{\mathrm{h}} 33^{\mathrm{m}} 34^{\mathrm{s}} .20, \delta=+30^{\circ} 32^{\prime} 11^{\prime \prime} .08$ (J2000), with an average $V$ magnitude of 18.86 , and an amplitude of variation in this band of about $0.11 \mathrm{mag}$. From the images it seems to be located in a small star cluster (see Fig. 5). Measured $V$ and $B$ magnitudes are given in Table 4.

The binary light curve in the $V$ band using the ephemeris of D99 (Fig. 4) shows two maxima and minima of different depth as expected for an ellipsoidal and X-ray heating light curve for a high mass XRB (HMXB) together with a doublesinusoidal fit to the data (average $V$ magnitude of $18.862 \pm$ 0.001 , amplitude $\left.(33 \pm 1) \times 10^{-3} \mathrm{mag}\right)$. The phase of the shallower minimum is shifted by $+0.0956 \pm 0.0028$ with respect to the eclipse center of D99 in the same direction as also indicated by the X-ray light curve (see Sect. 2.1). The double-sinusoidal fit is only a crude approximation to the data and the model parameters certainly are affected by non-uniform sampling of the optical light curve. The data suggest a deeper secondary minimum (around phase 0.6) which according to Tjemkes et al. (1986) might point at the effect of an accretion disk in the system. $V$ magnitude and $B-V$ color at $\mathrm{X}$-ray eclipse are 18.890 and -0.005 mag.

\section{Discussion}

\subsection{Improved ephemeris}

D99 determined the eclipse parameters for X-7 using in total $50 \mathrm{ks}$ and $325 \mathrm{ks}$ of archival ROSAT PSPC and HRI data, respectively, integrated over one satellite orbit ( $\sim 3 \mathrm{ks}$ time bins). The XMM-Newton and Chandra low and high state observations of $\mathrm{X}-7$ can naturally be explained by finding the source within and out of eclipse. Unfortunately, neither of the observations covered eclipse ingress or egress. However, from the XMM-Newton light curves we can deduce that the eclipse egress lasts $<0.02$ and the ingress $<0.16$ in phase (well within the parameter range given by D99). These values are only poorly determined due to gaps in the phase sampling over the orbit. More observations are needed to further constrain the binary ephemeris.

Nevertheless, we can restrict the time of eclipse egress to HJD $(2451760.935 \pm 0.035)$ assuming that it is constrained by the end of observation 0102640101 (when $\mathrm{X}-7$ is still in eclipse) and the beginning of observation 0141980501 (when, 261 binary orbits later, $X-7$ is already out of eclipse). With the eclipse shape parameters of D99 we then derive a mid-eclipse epoch of HJD (2 451 760.61 \pm 0.09$)$. The new X-ray mid-eclipse time corresponds to binary phase $0.07 \mathrm{in} \mathrm{Fig.} \mathrm{4.} \mathrm{Combining} \mathrm{this}$ result with the mid-eclipse epoch of D99 we get an improved orbital period for X-7 of $(3.45376 \pm 0.00021) \mathrm{d}$ well within the period errors given by $\mathrm{D} 99$.

\subsection{The optical companion}

The spectral classification of the optical companion can be deduced from the absolute optical magnitude and color 
Table 4. Optical light curve of the star identified with M 33 X-7. Magnitude with error in the $V$ or $B$ filter (Col. F) and heliocentric Julian date (HJD) of the observation are given.

\begin{tabular}{|c|c|c|c|c|c|}
\hline $\mathrm{F}$ & $\begin{array}{l}\text { HJD - } \\
2451400\end{array}$ & Mag & $\bar{F}$ & $\begin{array}{l}\text { HJD- } \\
2451400\end{array}$ & Mag \\
\hline V & 52.721458 & 18.854(4) & $B$ & 56.793912 & $18.887(4)$ \\
\hline$V$ & 52.730637 & $18.856(3)$ & V & 56.811296 & $18.884(4)$ \\
\hline V & 52.780012 & $18.852(3)$ & $B$ & 56.820741 & $18.873(4)$ \\
\hline V & 52.788819 & $18.849(4)$ & V & 56.829560 & $18.876(5)$ \\
\hline$B$ & 52.798183 & $18.852(3)$ & $B$ & 56.840301 & $18.865(4)$ \\
\hline V & 52.846146 & $18.861(4)$ & $V$ & 56.853021 & $18.871(4)$ \\
\hline V & 52.854931 & $18.861(4)$ & V & 56.877454 & $18.872(5)$ \\
\hline$V$ & 52.907662 & $18.858(6)$ & V & 56.887141 & $18.892(3)$ \\
\hline$B$ & 52.916632 & $18.856(5)$ & V & 57.674028 & $18.867(7)$ \\
\hline$V$ & 52.967407 & $18.867(5)$ & $B$ & 57.699340 & $18.829(8)$ \\
\hline$V$ & 52.976493 & $18.865(5)$ & V & 57.771528 & $18.848(5)$ \\
\hline$V$ & 53.005012 & $18.847(5)$ & V & 57.804664 & $18.851(5)$ \\
\hline V & 53.919248 & $18.841(4)$ & $B$ & 57.814074 & $18.838(4)$ \\
\hline$V$ & 54.676273 & $18.878(6)$ & V & 57.857789 & $18.830(4)$ \\
\hline V & 54.686343 & $18.881(5)$ & V & 57.866620 & $18.829(4)$ \\
\hline$B$ & 54.696771 & $18.866(3)$ & $B$ & 57.875891 & $18.831(4)$ \\
\hline$V$ & 54.705671 & $18.878(3)$ & V & 57.940764 & $18.814(5)$ \\
\hline$V$ & 54.720822 & $18.881(4)$ & $B$ & 57.950278 & $18.842(4)$ \\
\hline$B$ & 54.729942 & $18.855(3)$ & $B$ & 84.699097 & $18.873(3)$ \\
\hline$V$ & 54.738519 & $18.879(3)$ & V & 84.708727 & $18.884(3)$ \\
\hline$V$ & 54.777199 & $18.871(3)$ & V & 84.726852 & $18.890(4)$ \\
\hline$V$ & 54.791528 & $18.870(3)$ & V & 84.777234 & $18.866(4)$ \\
\hline$B$ & 54.800428 & $18.875(3)$ & $B$ & 84.785775 & $18.865(4)$ \\
\hline$V$ & 54.809769 & $18.873(4)$ & V & 84.794618 & $18.865(4)$ \\
\hline$V$ & 54.819468 & $18.874(3)$ & V & 84.849259 & $18.861(3)$ \\
\hline$V$ & 54.860521 & $18.884(4)$ & V & 84.883831 & $18.846(4)$ \\
\hline$B$ & 54.869780 & $18.897(4)$ & $B$ & 84.895440 & $18.844(3)$ \\
\hline$B$ & 54.878403 & $18.898(4)$ & V & 84.906539 & $18.855(4)$ \\
\hline$V$ & 54.906435 & $18.902(4)$ & V & 84.952697 & $18.850(7)$ \\
\hline$V$ & 54.915324 & $18.897(4)$ & $B$ & 86.634097 & $18.853(3)$ \\
\hline$V$ & 54.971019 & $18.914(4)$ & V & 86.643438 & $18.855(4)$ \\
\hline$B$ & 54.987986 & $18.923(4)$ & V & 86.727384 & $18.844(4)$ \\
\hline$V$ & 54.997303 & $18.924(4)$ & $B$ & 86.742824 & $18.837(4)$ \\
\hline$V$ & 55.753866 & $18.832(5)$ & V & 86.897975 & $18.821(6)$ \\
\hline$B$ & 55.762789 & $18.827(4)$ & V & 86.921400 & $18.814(6)$ \\
\hline$V$ & 55.861551 & $18.820(4)$ & V & 88.643009 & $18.841(4)$ \\
\hline$B$ & 55.870822 & $18.824(3)$ & $B$ & 88.652106 & $18.843(3)$ \\
\hline$V$ & 55.958808 & $18.813(4)$ & V & 88.664965 & $18.835(4)$ \\
\hline$V$ & 56.672176 & $18.888(4)$ & V & 88.728738 & $18.839(3)$ \\
\hline$B$ & 56.680891 & $18.895(3)$ & V & 88.741968 & $18.826(4)$ \\
\hline$V$ & 56.690012 & $18.884(4)$ & V & 88.750914 & $18.816(4)$ \\
\hline$B$ & 56.701400 & $18.883(3)$ & $B$ & 88.760104 & $18.845(2)$ \\
\hline$V$ & 56.715382 & $18.909(5)$ & $B$ & 88.780637 & $18.824(3)$ \\
\hline$B$ & 56.724583 & $18.906(4)$ & V & 88.878808 & $18.826(6)$ \\
\hline V & 56.739606 & $18.886(6)$ & V & 88.893958 & $18.830(7)$ \\
\hline$B$ & 56.748368 & $18.883(5)$ & V & 88.927685 & $18.813(7)$ \\
\hline$V$ & 56.757824 & $18.888(6)$ & V & 89.698310 & $18.912(4)$ \\
\hline$B$ & 56.766597 & $18.880(4)$ & V & 89.710938 & $18.912(3)$ \\
\hline$V$ & 56.775752 & $18.882(3)$ & V & 90.841655 & $18.838(5)$ \\
\hline$V$ & 56.786528 & $18.891(4)$ & V & 90.886076 & $18.835(6)$ \\
\hline
\end{tabular}

during X-ray eclipse when we see the optical surface that is mostly undisturbed by gravitational effects, an expected accretion disk and heating by the X-ray source. To derive the absolute magnitude the measured brightness has to be corrected for the distance $(-24.502$ mag for the assumed distance of $795 \mathrm{kpc}$ ) and for interstellar extinction, the color has to be corrected for reddening. These corrections can be estimated from the Galactic $N_{\mathrm{H}}$ in the direction of $\mathrm{X}-7$ as $A_{V \mathrm{Gal}}=0.36 \mathrm{mag}$ and $E(B-V)_{\mathrm{Gal}}=-0.12$ (Predehl \& Schmitt 1995). The $N_{\mathrm{H}}$ column depth of the M 33 disk in the direction of $\mathrm{X}-7$ is varying significantly on small scales. $\mathrm{X}-7$ is not located in one of the H I holes catalogued by Deul \& den Hartog (1990). The absorbing column within M 33 can be determined to $\sim 2.2 \times 10^{21} \mathrm{~cm}^{-2}$ from a $47 \times 93$ arcsec half power beam width H I map (Newton 1980). The absorbing columns derived from the spectral fits to the X-ray spectra indicate half or less than this absorbing column depending on the spectral model. Assuming that we see X-7 through half of this column and that the same conversion applies as used above, we get $A_{V \mathrm{M} 33}=0.62 \mathrm{mag}$ and $E(B-V)_{\mathrm{M} 33}=-0.21$. The companion star therefore should have an absolute $V$ magnitude of -6.0 to -6.6 and $(B-V)_{0}$ of -0.12 to -0.33 , which would correspond to a star of spectral typ B0I to O7I and masses $M_{2}$ of 25 to $35 M_{\odot}$ (see e.g. Aller et al. 1982). Optical companions of similar spectral type were proposed by D99 based on binary orbit and eclipse length, assuming a $1.4 M_{\odot}$ neutron star as the compact object.

\section{3. $M 33 X-7$, an eclipsing black hole $X R B$ ?}

We can use the mass of the optical companion as determined in the previous subsection and the mass ratios determined from binary orbit and eclipse length (see D99 and Paczyński 1971; Eggleton 1983) to determine the mass $M_{\mathrm{X}}$ of the compact object. For a binary inclination of $90^{\circ}$, the mass ratio $q\left(=M_{\mathrm{X}} / M_{2}\right)$ is 0.085 yielding $M_{\mathrm{X}}$ of $2.1-3.0 M_{\odot}$, significantly higher than expected masses of neutron stars. For an inclination of $70^{\circ}, q$ is 0.036 and $M_{\mathrm{X}}=0.9-1.2 M_{\odot}$ well in the range of typical neutron star masses. The inclination can not be constrained by our measurements and the mass estimates above do not exclude a neutron star for the compact object in the system.

Further information on the nature of the compact object may be infered from the X-ray spectrum. Before XMM-Newton and Chandra, the X-ray spectrum of X-7 was investigated using ROSAT and BeppoSAX observations. HP01 found that the $\mathrm{X}-7$ spectrum in the ROSAT band can be described by an absorbed power law $\left(N_{\mathrm{H}}=(1.9 \pm 0.9) \times\right.$ $10^{21} \mathrm{~cm}^{-2}$ and $\left.\Gamma=1.86 \pm 0.52\right)$. Thin thermal plasma models did not give acceptable fits. Parmar et al. (2001) reported that BeppoSAX detected $\mathrm{X}-7$ in the $2-8 \mathrm{keV}$ band. The spectrum could be described equally well by a power law $\left(N_{\mathrm{H}}<9.8 \times\right.$ $10^{22} \mathrm{~cm}^{-2}$ and photon index $\left.\Gamma=2.9_{-1.3}^{+1.7}\right)$ or a Bremsstrahlung model $\left(N_{\mathrm{H}}<11 \times 10^{22} \mathrm{~cm}^{-2}\right.$ and $\left.k T=3.7_{-2.4}^{+97}\right)$. If the absorption is fixed to $5.6 \times 10^{20} \mathrm{~cm}^{-2}$, the power law photon index is constrained to $1.7 \pm 0.6$ and the Bremsstrahlung temperature to $<3.7 \mathrm{keV}$. Keeping in mind the large uncertainties in the parameters, these results are consistent with the XMM-Newton and Chandra spectra reported for this persistent source in Sect. 2.3. In the following we therefore concentrate on these higher quality spectral results. 
Thermal Bremsstrahlung and disk blackbody models yield acceptable fits to the XMM-Newton and Chandra spectra (see Sect. 2.3). For the first model an absorbing column comparable to half the H I values at the position of X-7 within M 33 (see above) is needed to fit the $\mathrm{X}-7$ spectra. The second model only allows us to determine upper limits for the M 33 absorbing column in front of $\mathrm{X}-7$ which are smaller than half the M 33 column depth at that position. The X-7 X-ray spectra are steeper in the $2-10 \mathrm{keV}$ band than typical for HMXB (flat power law shapes with photon indices of $0.8<\Gamma<1.5$, White et al. 1983) and better resemble the spectra of persistent black hole XRBs like LMC X-1 and Cyg X-1 (see e.g. Cui et al. 2002).

Therefore, the spectral results, our unsuccessful search for $\mathrm{X}$-ray pulsations and the high mass estimate of the compact object, infered from the identified optical companion indicate a black hole as the compact object. These findings make M $33 \mathrm{X}-7$ a very interesting source for further investigation as it could be the first eclipsing HMXB with a black hole as the compact object.

\section{Conclusions}

XMM-Newton and Chandra observations of the persistent eclipsing HMXB M $33 \mathrm{X}-7$ allowed us to improve on the orbital period and investigate the $\mathrm{X}$-ray spectrum in unprecedented detail. No X-ray pulsations were detected. A special investigation of the optical variability of DIRECT data of the region revealed in the optical the orbital light curve of a high mass companion. X-ray and optical data point at a black hole as the compact object in the system. Optical spectroscopy and high sensitivity X-ray pulsation searches are needed to clarify the situation.

Acknowledgements. The XMM-Newton project is supported by the Bundesministerium für Bildung und Forschung / Deutsches Zentrum für Luft- und Raumfahrt (BMBF/DLR), the Max-Planck Society and the Heidenhain-Stiftung.

\section{References}

Alard, C. 2000, A\&AS, 144, 363

Alard, C., \& Lupton, R. H. 1998, ApJ, 503, 325

Aller, L. H., Appenzeller, I., Baschek, B., et al. (eds.) 1982, Landolt-Börnstein: Numerical Data and Functional Relationships in Science and Technology - New Series, Gruppe/Group 6 Astronomy and Astrophysics, Vol. 2 Schaifers/Voigt: Astronomy and Astrophysics / Astronomie und Astrophysik, Stars and Star Clusters / Sterne und Sternhaufen
Buccheri, R., Bennett, K., Bignami, G. F., et al. 1983, A\&A, 128, 245 Cui, W., Feng, Y. X., Zhang, S. N., et al. 2002, ApJ, 576, 357

Deul, E. R., \& den Hartog, R. H. 1990, A\&A, 229, 362

Dickey, J. M., \& Lockman, F. J. 1990, ARA\&A, 28, 215

Dubus, G., Charles, P. A., Long, K. S., Hakala, P. J., \& Kuulkers, E. 1999, MNRAS, 302, 731

Eggleton, P. P. 1983, ApJ, 268, 368

Gordon, S. M., Duric, N., Kirshner, R. P., Goss, W. M., \& Viallefond, F. 1999, ApJS, 120, 247

Haberl, F., \& Pietsch, W. 2001, A\&A, 373, 438

Haberl, F., \& Zavlin, V. E. 2002, A\&A, 391, 571

Humphreys, R. M., \& Sandage, A. 1980, ApJS, 44, 319

Jansen, F., Lumb, D., Altieri, B., et al. 2001, A\&A, 365, L1

Kaluzny, J., \& Udalski, A. 1992, Acta Astron., 42, 29

Larson, D. T., \& Schulman, E. 1997, AJ, 113, 618

Liu, Q. Z., van Paradijs, J., \& van den Heuvel, E. P. J. 2000, A\&AS, 147,25

Long, K. S., Dodorico, S., Charles, P. A., \& Dopita, M. A. 1981, ApJ, 246, L61

Macri, L. M., Stanek, K. Z., Sasselov, D. D., Krockenberger, M., \& Kaluzny, J. 2001, AJ, 121, 861

Markert, T. H., \& Rallis, A. D. 1983, ApJ, 275, 571

Mochejska, B. J., Kaluzny, J., Stanek, K. Z., Sasselov, D. D., \& Szentgyorgyi, A. H. 2001a, AJ, 121, 2032

Mochejska, B. J., Kaluzny, J., Stanek, K. Z., Sasselov, D. D., \& Szentgyorgyi, A. H. 2001b, AJ, 122, 2477

Mochejska, B. J., Kaluzny, J., Stanek, K. Z., Sasselov, D. D., \& Szentgyorgyi, A. H. 2001c, AJ, 121, 3284

Newton, K. 1980, MNRAS, 191, 615

Paczyński, B. 1971, ARA\&A, 9, 183

Parmar, A. N., Sidoli, L., Oosterbroek, T., et al. 2001, A\&A, 368, 420

Peres, G., Reale, F., Collura, A., \& Fabbiano, G. 1989, ApJ, 336, 140

Pietsch, W., Ehle, M., Haberl, F., Misanovic, Z., \& Trinchieri, G. 2003, Astron. Nach., 324, 85

Predehl, P., \& Schmitt, J. H. M. M. 1995, A\&A, 293, 889

Schulman, E., Bregman, J. N., Collura, A., Reale, F., \& Peres, G. 1993, ApJ, 418, L67

Schulman, E., Bregman, J. N., Collura, A., Reale, F., \& Peres, G. 1994, ApJ, 426, L55

Strüder, L., Briel, U., Dennerl, K., et al. 2001, A\&A, 365, L18

Tjemkes, S. A., van Paradijs, J., \& Zuiderwijk, E. J. 1986, A\&A, 154, 77

Trinchieri, G., Fabbiano, G., \& Peres, G. 1988a, ApJ, 325, 531

Trinchieri, G., Fabbiano, G., \& Peres, G. 1988b, ApJ, 329, 1037

Turner, M. J. L., Abbey, A., Arnaud, M., et al. 2001, A\&A, 365, L27

van den Bergh, S. 1991, PASP, 103, 609

Weisskopf, M. C., Tananbaum, H. D., Van Speybroeck, L. P., \& O’Dell, S. L. 2000, Proc. SPIE, 4012, 2

White, N. E., Swank, J. H., \& Holt, S. S. 1983, ApJ, 270, 711 